International Mathematical Forum, Vol. 8, 2013, no. 27, 1323 - 1336

HIKARI Ltd, www.m-hikari.com

http://dx.doi.org/10.12988/imf.2013.3473

\title{
An M/G/1 Queue with Two-Stage Heterogeneous Service and Single Working Vacation
}

\author{
K. Santhi and S. Pazhani Bala Murugan \\ Mathematics Section, Faculty of Engineering and Technology \\ Annamalai University, Annamalainagar-608 002, India \\ santhimano3169@gmail.com, spbmaths@yahoo.co.in
}

Copyright (c) 2013 K. Santhi and S. Pazhani Bala Murugan. This is an open access article distributed under the Creative Commons Attribution License, which permits unrestricted use, distribution, and reproduction in any medium, provided the original work is properly cited.

\begin{abstract}
We study an M/G/1 queue with two stages of heterogeneous service and single working vacation.Using supplementary variable approach we derive the probability generating function for the number of customers and the average number of customers in the system. Some special cases of interest are discussed.
\end{abstract}

Mathematics Subject Classification: 60K25, 60K30

Keywords: Poisson arrivals, Two-Stage Heterogeneous Service, Working Vacation, Supplementary Variable Technique 


\section{Introduction}

The $M / G / 1$ queue with vacation time has been studied by number of authors. To mention a few references we would name Cooper (1981), Levy et.al. (1975), Yukata Baba (1986), Teghem (1986) and Doshi (1990). Recently a class of semi-vacation policies has been introduced by Servi and Finn. Such a vacation is called working vacation(WV). The server works at a lower rate rather than completely stops service during a vacation. Servi and Finn (2002) studied an $M / M / 1$ queue with multiple working vacation and obtained the probability generating function for the number of customers in the system and the waiting time distribution. Some other notable works were done by $\mathrm{Wu}$ and Takagi (2006), Tian et.al. (2008) and Afthab Begum (2011).

In many queueing situations all arriving customers require the main service and only some of them may require the subsidiary service provided by the server. Madan (2000) investigated an $M / G / 1$ queueing system with second optional service. The single server vacation queueing models with second optional service were analyzed by many authors including Kalyanaraman et.al. (2008) and Thangaraj (2010).

In this paper we study an $M / G / 1$ queue with Two-stage Heterogeneous service and single working vacation(SWV). The organization of the paper is as follows. In section 2 we described the model. In section 3 we obtained the steady state probability generating function. In section 4 the performance measures are obtained and in section 5 particular cases have been discussed.

\section{The Model description}

We consider a single server queueing system in which customers arrive according to a Poisson process with mean arrival rate $\lambda(>0)$. The service discipline is FCFS. Each arriving customer undergoes the first essential stage (FES) of service which has general distribution with the distribution function $S_{b_{1}}(x)$, the probability density function $s_{b_{1}}(x)$ and the LaplaceStieltjes transform[LST] $S_{b_{1}}^{*}(\theta)$ where $S_{b_{1}}$ is the service time of the first stage service. After the completion of FES of service the customer may opt for the second optional stage (SOS) of service with probability $p$ or the customer 
may leave the system with out taking the SOS of service with probability $q(p+q=1)$.

The SOS of service also follows the general distribution with the distribution function $S_{b_{2}}(x)$, the probability density function $s_{b_{2}}(x)$ and $S_{b_{2}}^{*}(\theta)$ be the LST of $S_{b_{2}}(x)$, where $S_{b_{2}}$ is the service time of the second stage service.

Whenever the system becomes empty at a service completion instant the server starts working vacation and the duration of the vacation time follows an exponential distribution with rate $\eta$. At a vacation completion instant, if there are customers in the system the server will start a new busy period. Otherwise he waits until a customer arrive. This type of vacation policy is called single working vacation. During the working vacation period the server also provides two stages of service. The first essential stage of service time $S_{v_{1}}$ of a typical customer follows a general distribution with the distribution function $S_{v_{1}}(x)\left[s_{v_{1}}(x)\right.$ the probability density function and $S_{v_{1}}^{*}(\theta)$, the LST] and the SOS of service time $S_{v_{2}}$ also follows a general distribution with the distribution function $S_{v_{2}}(x)\left[s_{v_{2}}(x)\right.$ the probability density function and $S_{v_{2}}^{*}(\theta)$, the LST].

Further, it is noted that the service interrupted at the end of a vacation is lost and it is restarted with a different distribution at the beginning of the following service period. Inter arrival times, service times and working vacation times are mutually independent of each other.

\section{The System Size Distribution at a Random Epoch}

The system size distribution at an arbitrary time will be treated by the supplementary variable technique. That is, from the joint distribution of the queue length and the remaining service time of the customer in service if the server is busy, or the remaining service time of the customer if the server is on WV.

Assuming that the system is in steady state condition. Let us define the following random variables.

$N(t)$ - the system size at time $t$.

$S_{b_{1}}^{0}(t)$ - the remaining service time for the FES of service in not WV period. 
$S_{b_{2}}^{0}(t)$ - the remaining service time for the SOS of service in not WV period. $S_{v_{1}}^{0}(t)$ - the remaining service time for the FES of service in WV period. $S_{v_{2}}^{0}(t)$ - the remaining service time for the SOS of service in WV period.

$Y(t)= \begin{cases}0 & \text { if the server is idle on WV period } \\ 1 & \text { if the server is idle on not WV period } \\ 2 & \text { if the server is busy for giving FES of service in not WV period } \\ 3 & \text { if the server is busy for giving SOS of service in not WV period } \\ 4 & \text { if the server is busy for giving FES of service in WV period } \\ 5 & \text { if the server is busy for giving SOS of service in WV period }\end{cases}$

so that the supplementary variables $S_{b_{1}}^{0}(t), S_{b_{2}}^{0}(t), S_{v_{1}}^{0}(t)$ and $S_{v_{2}}^{0}(t)$ are introduced in order to obtain bivariate Markov Process $\{N(t), \partial(t)\}$, where

$$
\partial(t)= \begin{cases}S_{b_{1}}^{0}(t) & \text { if } Y(t)=2 \\ S_{b_{2}}^{0}(t) & \text { if } Y(t)=3 \\ S_{v_{1}}^{0}(t) & \text { if } Y(t)=4 \\ S_{v_{2}}^{0}(t) & \text { if } Y(t)=5\end{cases}
$$

We define the following limiting probabilities:

$Q_{0}=\lim _{t \rightarrow \infty} \operatorname{Pr}\{N(t)=0, Y(t)=0\}$

$P_{0}=\lim _{t \rightarrow \infty} \operatorname{Pr}\{N(t)=0, Y(t)=1\}$

$Q_{n, i}(x)=\lim _{t \rightarrow \infty} \operatorname{Pr}\left\{N(t)=n, Y(t)=\{4,5\}, x<S_{v_{i}}^{0}(t) \leq x+d x\right\}, i=1,2 ; n \geq$ 1.

$P_{n, i}(x)=\lim _{t \rightarrow \infty} \operatorname{Pr}\left\{N(t)=n, Y(t)=\{2,3\}, x<S_{b_{i}}^{0}(t) \leq x+d x\right\}, i=1,2 ; n \geq 1$. By considering the steady state, we have the following system of the differential difference equations.

$$
\begin{aligned}
(\lambda+\eta) Q_{0} & =q P_{1,1}(0)+P_{1,2}(0)+q Q_{1,1}(0)+Q_{1,2}(0) \\
-\frac{d}{d x} Q_{1,1}(x) & =-(\lambda+\eta) Q_{1,1}(x)+q Q_{2,1}(0) s_{v_{1}}(x)+Q_{2,2}(0) s_{v_{1}}(x)+\lambda Q_{0} s_{v_{1}}(x)(2) \\
-\frac{d}{d x} Q_{n, 1}(x) & =-(\lambda+\eta) Q_{n, 1}(x)+q Q_{n+1,1}(0) s_{v_{1}}(x)+Q_{n+1,2}(0) s_{v_{1}}(x)+ \\
\lambda Q_{n-1,1}(x) &
\end{aligned}
$$




$$
\begin{gathered}
-\frac{d}{d x} Q_{1,2}(x)=-(\lambda+\eta) Q_{1,2}(x)+p Q_{1,1}(0) s_{v_{2}}(x) \\
-\frac{d}{d x} Q_{n, 2}(x)=-(\lambda+\eta) Q_{n, 2}(x)+p Q_{n, 1}(0) s_{v_{2}}(x)+\lambda Q_{n-1,2}(x) ; n>1 \\
\lambda P_{0}=\eta Q_{0} \\
\quad+\eta s_{b_{1}}(x) \int_{0}^{\infty} Q_{1,1}(y) d y+\lambda P_{0} s_{b_{1}}(x) \\
\quad+\eta s_{b_{1}}(x) \int_{0}^{\infty} Q_{n, 1}(y) d y+\lambda P_{n-1,1}(x) ; n>1 \\
-\frac{d}{d x} P_{n, 1}(x)=-\lambda P_{n, 1}(x)+q P_{n+1,1}(0) s_{b_{1}}(x)+P_{n+1,2}(0) s_{b_{1}}(x) \\
-\frac{d}{d x} P_{1,2}(x)=-\lambda P_{1,2}(x)+p P_{1,1}(0) s_{b_{2}}(x)+\eta s_{b_{2}}(x) \int_{0}^{\infty} Q_{1,2}(y) d y \\
-\frac{d}{d x} P_{n, 2}(x)=-\lambda P_{n, 2}(x)+p P_{n, 1}(0) s_{b_{2}}(x) \\
+\eta s_{b_{2}}(x) \int_{0}^{\infty} Q_{n, 2}(y) d y+\lambda P_{n-1,2}(x) ; n>1
\end{gathered}
$$

We define Laplace Stieltjes transforms and probability generating functions as follows,

$$
\begin{array}{ll}
S_{b_{i}}^{*}(\theta)=\int_{0}^{\infty} e^{-\theta x} s_{b_{i}}(x) d x ; & i=1,2 \\
S_{v_{i}}^{*}(\theta)=\int_{0}^{\infty} e^{-\theta x} s_{v_{i}}(x) d x ; & i=1,2 \\
Q_{n, i}^{*}(\theta)=\int_{0}^{\infty} e^{-\theta x} Q_{n, i}(x) d x ; & i=1,2 \\
P_{n, i}^{*}(\theta)=\int_{0}^{\infty} e^{-\theta x} P_{n, i}(x) d x ; & i=1,2 \\
Q_{i}^{*}(z, \theta)=\sum_{n=1}^{\infty} z^{n} Q_{n, i}^{*}(\theta) ; & i=1,2 \\
Q_{i}(z, 0)=\sum_{n=1}^{\infty} z^{n} Q_{n, i}(0) ; & i=1,2 \\
P_{i}^{*}(z, \theta)=\sum_{n=1}^{\infty} z^{n} P_{n, i}^{*}(\theta) ; & i=1,2 \\
P_{i}(z, 0)=\sum_{n=1}^{\infty} z^{n} P_{n, i}(0) ; & i=1,2
\end{array}
$$

Taking the LST of (2) to (5) and (7) to (10) we have

$\theta Q_{1,1}^{*}(\theta)-Q_{1,1}(0)=(\lambda+\eta) Q_{1,1}^{*}(\theta)-q Q_{2,1}(0) S_{v_{1}}^{*}(\theta)-Q_{2,2}(0) S_{v_{1}}^{*}(\theta)-\lambda Q_{0} S_{v_{1}}^{*}(\theta)$ 
$\theta Q_{n, 1}^{*}(\theta)-Q_{n, 1}(0)=(\lambda+\eta) Q_{n, 1}^{*}(\theta)-q Q_{n+1,1}(0) S_{v_{1}}^{*}(\theta)-Q_{n+1,2}(0) S_{v_{1}}^{*}(\theta)-$ $\lambda Q_{n-1,1}^{*}(\theta)$;

$\theta Q_{1,2}^{*}(\theta)-Q_{1,2}(0)=(\lambda+\eta) Q_{1,2}^{*}(\theta)-p Q_{1,1}(0) S_{v_{2}}^{*}(\theta)$

$\theta Q_{n, 2}^{*}(\theta)-Q_{n, 2}(0)=(\lambda+\eta) Q_{n, 2}^{*}(\theta)-p Q_{n, 1}(0) S_{v_{2}}^{*}(\theta)-\lambda Q_{n-1,2}^{*}(\theta)$;

$$
\begin{aligned}
& \theta P_{1,1}^{*}(\theta)-P_{1,1}(0)=\lambda P_{1,1}^{*}(\theta)-q P_{2,1}(0) S_{b_{1}}^{*}(\theta) \\
& -P_{2,2}(0) S_{b_{1}}^{*}(\theta)-\lambda P_{0} S_{b_{1}}^{*}(\theta)-\eta S_{b_{1}}^{*}(\theta) \int_{0}^{\infty} Q_{1,1}(y) d y \\
& \theta P_{n, 1}^{*}(\theta)-P_{n, 1}(0)=\lambda P_{n, 1}^{*}(\theta)-q P_{n+1,1}(0) S_{b_{1}}^{*}(\theta)-P_{n+1,2}(0) S_{b_{1}}^{*}(\theta) \\
& -\eta S_{b_{1}}^{*}(\theta) \int_{0}^{\infty} Q_{n, 1}(y) d y-\lambda P_{n-1,1}^{*}(\theta) ; \quad n>1 \\
& \theta P_{1,2}^{*}(\theta)-P_{1,2}(0)=\lambda P_{1,2}^{*}(\theta)-p P_{1,1}(0) S_{b_{2}}^{*}(\theta)-\eta S_{b_{2}}^{*}(\theta) \int_{0}^{\infty} Q_{1,2}(y) d y \\
& \theta P_{n, 2}^{*}(\theta)-P_{n, 2}(0)=\lambda P_{n, 2}^{*}(\theta)-p P_{n, 1}(0) S_{b_{2}}^{*}(\theta) \\
& -\eta S_{b_{2}}^{*}(\theta) \int_{0}^{\infty} Q_{n, 2}(y) d y-\lambda P_{n-1,2}^{*}(\theta) ; \quad n>1
\end{aligned}
$$

$z^{n}$ times (12) summing over $n$ from 2 to $\infty$ is added up with $z$ times (11) we get

$[\theta-(\lambda-\lambda z+\eta)] Q_{1}^{*}(z, \theta)=$

$$
Q_{1}(z, 0)\left[1-\frac{q}{z} S_{v_{1}}^{*}(\theta)\right]+S_{v_{1}}^{*}(\theta)\left[q Q_{1,1}(0)-\frac{Q_{2}(z, 0)}{z}+Q_{1,2}(0)-\lambda Q_{0} z\right]
$$

$z^{n}$ times (14) summing over $n$ from 2 to $\infty$ is added up with $z$ times (13) we get

$[\theta-(\lambda-\lambda z+\eta)] Q_{2}^{*}(z, \theta)=Q_{2}(z, 0)-p S_{v_{2}}^{*}(\theta) Q_{1}(z, 0)$

Inserting $\theta=(\lambda-\lambda z+\eta)$ in (19) and (20) we get

$Q_{1}(z, 0)=\left[\frac{z S_{v_{1}}^{*}(\lambda-\lambda z+\eta)\left[\lambda Q_{0} z-q Q_{1,1}(0)-Q_{1,2}(0)\right]}{z-(1-p) S_{v_{1}}^{*}(\lambda-\lambda z+\eta)-p S_{v_{1}}^{*}(\lambda-\lambda z+\eta) S_{v_{2}}^{*}(\lambda-\lambda z+\eta)}\right]$

The denominator of the above equation has a unique root $z_{1}$ in $(0,1)$. Therefore

$q Q_{1,1}(0)+Q_{1,2}(0)=\lambda Q_{0} z_{1}$

Substituting this in (21) we have

$Q_{1}(z, 0)=\left[\frac{\lambda z Q_{0} S_{v_{1}}^{*}(\lambda-\lambda z+\eta)\left(z-z_{1}\right)}{z-(1-p) S_{v_{1}}^{*}(\lambda-\lambda z+\eta)-p S_{v_{1}}^{*}(\lambda-\lambda z+\eta) S_{v_{2}}^{*}(\lambda-\lambda z+\eta)}\right]$ 
and

$Q_{2}(z, 0)=\left[\frac{p S_{v_{2}}^{*}(\lambda-\lambda z+\eta) \lambda z Q_{0}\left(z-z_{1}\right) S_{v_{1}}^{*}(\lambda-\lambda z+\eta)}{z-(1-p) S_{v_{1}}^{*}(\lambda-\lambda z+\eta)-p S_{v_{1}}^{*}(\lambda-\lambda z+\eta) S_{v_{2}}^{*}(\lambda-\lambda z+\eta)}\right]$

(23) Substituting (22) and (23) in (19) and (20) and putting $\theta=0$, we have

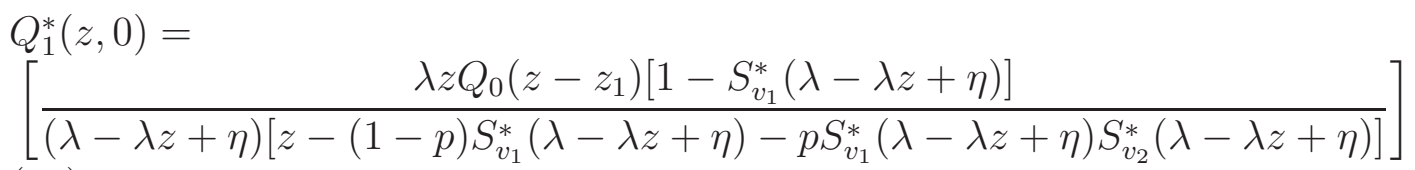

$\left[\frac{Q_{2}^{*}(z, 0)=}{p \lambda Q_{0} z\left(z-z_{1}\right) S_{v_{1}}^{*}(\lambda-\lambda z+\eta)\left[1-S_{v_{2}}^{*}(\lambda-\lambda z+\eta)\right]}\left[\frac{1}{(\lambda-\lambda z+\eta)\left[z-(1-p) S_{v_{1}}^{*}(\lambda-\lambda z+\eta)-p S_{v_{1}}^{*}(\lambda-\lambda z+\eta) S_{v_{2}}^{*}(\lambda-\lambda z+\eta)\right]}\right]\right.$

$z^{n}$ times (16) summing over $n$ from 2 to $\infty$ is added up with $z$ times (15) we get

$$
\begin{aligned}
& {[\theta-(\lambda-\lambda z)] P_{1}^{*}(z, \theta) }=P_{1}(z, 0)\left[\frac{z-q S_{b_{1}}^{*}(\theta)}{z}\right] \\
&-S_{b_{1}}^{*}(\theta)\left[\eta Q_{1}^{*}(z, 0)+\lambda z P_{0}-q P_{1,1}(0)+\frac{P_{2}(z, 0)}{z}-P_{1,2}(0)\right]
\end{aligned}
$$

$z^{n}$ times (18) summing over $n$ from 2 to $\infty$ is added up with $z$ times (17) we get

$[\theta-(\lambda-\lambda z)] P_{2}^{*}(z, \theta)=P_{2}(z, 0)-p S_{b_{2}}^{*}(\theta) P_{1}(z, 0)-\eta S_{b_{2}}^{*}(\theta) Q_{2}^{*}(z, 0)$

Inserting $\theta=(\lambda-\lambda z)$ in (26) and (27) and also substituting $q P_{1,1}(0)+P_{1,2}(0)=$ $Q_{0}\left[\lambda\left(1-z_{1}\right)+\eta\right]$ in (26) we get $P_{1}(z, 0)=$

$\left[\frac{S_{b_{1}}^{*}(\lambda-\lambda z)\left[\eta z Q_{1}^{*}(z, 0)+\eta S_{b_{2}}^{*}(\lambda-\lambda z) Q_{2}^{*}(z, 0)-\lambda z Q_{0}\left(1-z_{1}\right)-\eta z(1-z) Q_{0}\right]}{z-(1-p) S_{b_{1}}^{*}(\lambda-\lambda z)-p S_{b_{1}}^{*}(\lambda-\lambda z) S_{b_{2}}^{*}(\lambda-\lambda z)}\right]$

and

$$
\begin{array}{r}
P_{2}(z, 0)=\left[S _ { b _ { 2 } } ^ { * } ( \lambda - \lambda z ) \left\{p z S_{b_{1}}^{*}(\lambda-\lambda z)\left[\eta Q_{1}^{*}(z, 0)-\eta(1-z) Q_{0}-\lambda Q_{0}\left(1-z_{1}\right)\right]\right.\right. \\
\left.\left.+\eta Q_{2}^{*}(z, 0)\left[z-(1-p) S_{b_{1}}^{*}(\lambda-\lambda z)\right]\right\}\right]
\end{array}
$$

$\overline{z-(1-p) S_{b_{1}}^{*}(\lambda-\lambda z)-p S_{b_{1}}^{*}(\lambda-\lambda z) S_{b_{2}}^{*}(\lambda-\lambda z)}$ 
Substituting (28) and (29) in (26) and (27) and inserting $\theta=0$, we get

$$
\begin{aligned}
& P_{1}^{*}(z, 0)=\frac{Q_{0}}{D_{1}(z) D_{2}(z)}\left\{z [ 1 - S _ { b _ { 1 } } ^ { * } ( \lambda - \lambda z ) ] \left\{\lambda \eta z\left(z-z_{1}\right)\left[1-S_{v_{1}}^{*}(\lambda-\lambda z+\eta)\right]\right.\right. \\
& +\lambda p \eta S_{b_{2}}^{*}(\lambda-\lambda z)\left(z-z_{1}\right) S_{v_{1}}^{*}(\lambda-\lambda z+\eta)\left(1-S_{v_{2}}^{*}(\lambda-\right. \\
& \lambda z+\eta)) \\
& -\left[\eta(1-z)+\lambda\left(1-z_{1}\right)\right](\lambda-\lambda z+\eta) \\
& \times\left[z-(1-p) S_{v_{1}}^{*}(\lambda-\lambda z+\eta)-p S_{v_{1}}^{*}(\lambda-\lambda z+\eta)\right. \\
& \left.\left.\left.S_{v_{2}}^{*}(\lambda-\lambda z+\eta)\right]\right\}\right\} \\
& P_{2}^{*}(z, 0)=\frac{Q_{0}}{D_{1}(z) D_{2}(z)}\left\{p z [ 1 - S _ { b _ { 2 } } ^ { * } ( \lambda - \lambda z ) ] \left\{\lambda \eta\left(z-z_{1}\right) S_{v_{1}}^{*}(\lambda-\lambda z+\eta)\right.\right. \\
& \times\left(1-S_{v_{2}}^{*}(\lambda-\lambda z+\eta)\right)\left(z-(1-p) S_{b_{1}}^{*}(\lambda-\lambda z)\right) \\
& +\lambda \eta z\left(z-z_{1}\right) S_{b_{1}}^{*}(\lambda-\lambda z)\left(1-S_{v_{1}}^{*}(\lambda-\lambda z+\eta)\right) \\
& -\left[\eta(1-z)+\lambda\left(1-z_{1}\right)\right](\lambda-\lambda z+\eta) S_{b_{1}}^{*}(\lambda-\lambda z) \\
& \times\left[z-(1-p) S_{v_{1}}^{*}(\lambda-\lambda z+\eta)\right. \\
& \left.\left.\left.-p S_{v_{1}}^{*}(\lambda-\lambda z+\eta) S_{v_{2}}^{*}(\lambda-\lambda z+\eta)\right]\right\}\right\}
\end{aligned}
$$

where

$D_{1}(z)=(\lambda-\lambda z)\left[z-(1-p) S_{v_{1}}^{*}(\lambda-\lambda z+\eta)-p S_{v_{1}}^{*}(\lambda-\lambda z+\eta) S_{v_{2}}^{*}(\lambda-\lambda z+\eta)\right]$

$D_{2}(z)=(\lambda-\lambda z+\eta)\left[z-(1-p) S_{b_{1}}^{*}(\lambda-\lambda z)-p S_{b_{1}}^{*}(\lambda-\lambda z) S_{b_{2}}^{*}(\lambda-\lambda z)\right]$

We define

$$
P_{B}(z)=P_{1}^{*}(z, 0)+P_{2}^{*}(z, 0)+P_{0}
$$

as the probability generating function for the number of customers in the system when the server is on not WV period and

$$
P_{V}(z)=Q_{1}^{*}(z, 0)+Q_{2}^{*}(z, 0)+Q_{0}
$$

as the probability generating function for the number of customers in the system when the server is on WV period then

$$
P(z)=P_{B}(z)+P_{V}(z)
$$

as the probability generating function for the number of customers in the system. We shall now use the normalizing condition $P(1)=1$ to determine the only unknown $Q_{0}$, which appears in (36). Substituting $z=1$ in (36) and using L'hospital's rule we obtain on simplification 


$$
Q_{0}=\frac{\left(1-\rho_{b}\right)}{\left[\frac{\eta}{\lambda}+\frac{\left(\lambda-\lambda z_{1}+\eta\right)}{\eta}-\frac{\lambda\left(1-z_{1}\right) S_{v_{1}}^{*}(\eta)\left[E\left(S_{b_{1}}\right)+p S_{v_{2}}^{*}(\eta) E\left(S_{b_{2}}\right)\right]}{1-(1-p) S_{v_{1}}^{*}(\eta)-p S_{v_{1}}^{*}(\eta) S_{v_{2}}^{*}(\eta)}\right]}
$$

where $\rho_{b}=\lambda\left[E\left(S_{b_{1}}\right)+p E\left(S_{b_{2}}\right)\right], E\left(S_{b_{1}}\right)$ and $E\left(S_{b_{2}}\right)$ are the mean service times of stage 1 and stage 2 respectively. From (37) we obtain the system stability condition,

$$
\rho_{b}<1
$$

\section{Performance Measures}

Mean System Length

Let $L_{v}$ and $L_{b}$ denote the mean system size during the working vacation and not working vacation period respectively. Then

$$
\begin{aligned}
& L_{v}=\frac{d}{d z} P_{V}(z) \text { at } z=1 \\
& =\frac{d}{d z}\left\{\frac{\left[N_{1}(z)+N_{2}(z)\right]}{D(z)} \lambda Q_{0}\right\} \text { at } z=1 \text { where } \\
& N_{1}(z)=z\left(z-z_{1}\right)\left[1-S_{v_{1}}^{*}(\lambda-\lambda z+\eta)\right] \\
& N_{2}(z)=p z\left(z-z_{1}\right) S_{v_{1}}^{*}(\lambda-\lambda z+\eta)\left[1-S_{v_{2}}^{*}(\lambda-\lambda z+\eta)\right] \\
& D(z)=(\lambda-\lambda z+\eta)\left[z-(1-p) S_{v_{1}}^{*}(\lambda-\lambda z+\eta)-p S_{v_{1}}^{*}(\lambda-\lambda z+\eta) S_{v_{2}}^{*}(\lambda-\lambda z+\eta)\right] \\
& L_{v}=\frac{\lambda Q_{0}\left\{D(1)\left[N_{1}^{\prime}(1)+N_{2}^{\prime}(1)\right]-D^{\prime}(1)\left[N_{1}(1)+N_{2}(1)\right]\right\}}{(D(1))^{2}} \text { where } \\
& N_{1}(1)=\left(1-z_{1}\right)\left(1-S_{v_{1}}^{*}(\eta)\right) \\
& N_{2}(1)=p\left(1-z_{1}\right) S_{v_{1}}^{*}(\eta)\left(1-S_{v_{2}}^{*}(\eta)\right) \\
& N_{1}^{\prime}(1)=\left(1-S_{v_{1}}^{*}(\eta)\right)+\left(1-z_{1}\right)\left(1-s_{v_{1}}^{*}(\eta)+\lambda S_{v_{1}}^{*^{\prime}}(\eta)\right) \\
& N_{2}^{\prime}(1)=p\left\{S_{v_{1}}^{*}(\eta)\left(1-S_{v_{2}}^{*}(\eta)\right)+\left(1-z_{1}\right)\left\{S_{v_{1}}^{*}(\eta)\left(1-S_{v_{2}}^{*}(\eta)\right)-\lambda S_{v_{1}}^{*^{\prime}}(\eta)\right.\right. \\
& \left.\left.+\lambda\left(S_{v_{1}}^{*^{\prime}}(\eta) S_{v_{2}}^{*}(\eta)+S_{v_{1}}^{*}(\eta) S_{v_{2}}^{*^{\prime}}(\eta)\right)\right\}\right\} \\
& D(1)=\eta\left\{1-(1-p) S_{v_{1}}^{*}(\eta)-p S_{v_{1}}^{*}(\eta) S_{v_{2}}^{*}(\eta)\right\} \\
& D^{\prime}(1)=-\lambda\left[1-(1-p) S_{v_{1}}^{*}(\eta)-p S_{v_{1}}^{*}(\eta) S_{v_{2}}^{*}(\eta)\right] \\
& +\eta\left[1+\lambda(1-p) S_{v_{1}}^{*^{\prime}}(\eta)+p \lambda\left(S_{v_{1}}^{*^{\prime}}(\eta) S_{v_{2}}^{*}(\eta)+S_{v_{1}}^{*}(\eta) S_{v_{2}}^{*^{\prime}}(\eta)\right)\right]
\end{aligned}
$$




$$
\begin{aligned}
L_{b}= & \frac{d}{d z} P_{B}(z) \text { at } z=1 \\
= & \frac{d}{d z}\left\{\frac{\left[N_{3}(z) N_{4}(z)+N_{5}(z) N_{6}(z)\right]}{D_{1}(z) D_{2}(z)} Q_{0}\right\} \text { at } z=1 \text { where } \\
N_{3}(z)= & z\left[1-S_{b_{1}}^{*}(\lambda-\lambda z)\right] \\
N_{4}(z)= & \lambda \eta z\left(z-z_{1}\right)\left[1-S_{v_{1}}^{*}(\lambda-\lambda z+\eta)\right] \\
& +\lambda p \eta S_{b_{2}}^{*}(\lambda-\lambda z)\left(z-z_{1}\right) S_{v_{1}}^{*}(\lambda-\lambda z+\eta)\left(1-S_{v_{2}}^{*}(\lambda-\lambda z+\eta)\right) \\
& \quad-\left[\eta(1-z)+\lambda\left(1-z_{1}\right)\right](\lambda-\lambda z+\eta) \\
& \left.\quad \times\left[z-(1-p) S_{v_{1}}^{*}(\lambda-\lambda z+\eta)-p S_{v_{1}}^{*}(\lambda-\lambda z+\eta) S_{v_{2}}^{*}(\lambda-\lambda z+\eta)\right]\right\} \\
N_{5}(z)= & p z\left[1-S_{b_{2}}^{*}(\lambda-\lambda z)\right] \\
N_{6}(z)= & \left\{\lambda \eta\left(z-z_{1}\right) S_{v_{1}}^{*}(\lambda-\lambda z+\eta)\left(1-S_{v_{2}}^{*}(\lambda-\lambda z+\eta)\right)\left(z-(1-p) S_{b_{1}}^{*}(\lambda-\lambda z)\right)\right. \\
\quad & \quad+\lambda \eta z\left(z-z_{1}\right) S_{b_{1}}^{*}(\lambda-\lambda z)\left(1-S_{v_{1}}^{*}(\lambda-\lambda z+\eta)\right) \\
& \quad-\left[\eta(1-z)+\lambda\left(1-z_{1}\right)\right](\lambda-\lambda z+\eta) S_{b_{1}}^{*}(\lambda-\lambda z) \\
\quad & \left.\quad \times\left[z-(1-p) S_{v_{1}}^{*}(\lambda-\lambda z+\eta)-p S_{v_{1}}^{*}(\lambda-\lambda z+\eta) S_{v_{2}}^{*}(\lambda-\lambda z+\eta)\right]\right\}
\end{aligned}
$$

$D_{1}(z)$ and $D_{2}(z)$ are given respectively in equations (32) and (33)

Therefore

$$
L_{b}=\frac{Q_{0}}{2\left[D_{1}^{\prime}(1)\right]^{2}\left[D_{2}^{\prime}(1)\right]^{2}}\left\{\begin{array}{r}
D_{1}^{\prime}(1) D_{2}^{\prime}(1)\left[N_{3}^{\prime \prime}(1) N_{4}^{\prime}(1)+N_{3}^{\prime}(1) N_{4}^{\prime \prime}(1)\right. \\
+ \\
\left.+N_{5}^{\prime \prime}(1) N_{6}^{\prime}(1)+N_{5}^{\prime}(1) N_{6}^{\prime \prime}(1)\right]-\left(N_{3}^{\prime}(1) N_{4}^{\prime}(1)\right. \\
\left.\left.+N_{5}^{\prime}(1) N_{6}^{\prime}(1)\right)\left[D_{1}^{\prime \prime}(1) D_{2}^{\prime}(1)+D_{1}^{\prime}(1) D_{2}^{\prime \prime}(1)\right]\right\}
\end{array}\right.
$$

where

$$
\begin{aligned}
& N_{3}^{\prime}(1)=-\lambda E\left(S_{b_{1}}\right) \\
& N_{3}^{\prime \prime}(1)=-\left[2 \lambda E\left(S_{b_{1}}\right)+\lambda^{2} E\left(S_{b_{1}}^{2}\right)\right] \\
& N_{4}^{\prime}(1)=\eta \lambda\left\{p S_{v_{1}}^{*}(\eta)\left(1-S_{v_{2}}^{*}(\eta)\right)\left(1+\lambda\left(1-z_{1}\right) E\left(S_{b_{2}}\right)\right)+\left(1-S_{v_{1}}^{*}(\eta)\right)-(1-\right. \\
& \begin{aligned}
\left.\left.z_{1}\right) S_{v_{1}}^{*}(\eta)\right\} \\
\quad+\left(\eta^{2}+\lambda^{2}\left(1-z_{1}\right)\right)\left[1-(1-p) S_{v_{1}}^{*}(\eta)-p S_{v_{1}}^{*}(\eta) S_{v_{2}}^{*}(\eta)\right] \\
N_{4}^{\prime \prime}(1)=2 \eta \lambda\left\{1-S_{v_{1}}^{*}(\eta)+p \lambda E\left(S_{b_{2}}\right) S_{v_{1}}^{*}(\eta)\left(1-S_{v_{2}}^{*}(\eta)\right)\right. \\
\left.\quad+(1-p) \lambda S_{v_{1}}^{*^{\prime}}(\eta)+p \lambda\left(S_{v_{1}}^{*^{\prime}}(\eta) S_{v_{2}}^{*}(\eta)+S_{v_{1}}^{*}(\eta) S_{v_{2}}^{*^{\prime}}(\eta)\right)\right\} \\
\quad+\eta \lambda\left(1-z_{1}\right)\left\{p \lambda^{2} E\left(S_{b_{2}}^{2}\right) S_{v_{1}}^{*}(\eta)\left(1-S_{v_{2}}^{*}(\eta)\right)-2 p \lambda^{2} E\left(S_{b_{2}}\right) S_{v_{1}}^{*^{\prime}}(\eta)\right. \\
\left.\quad+2 p \lambda^{2} E\left(S_{b_{2}}\right)\left(S_{v_{1}}^{*^{\prime}}(\eta) S_{v_{2}}^{*}(\eta)+S_{v_{1}}^{*}(\eta) S_{v_{2}}^{*^{\prime}}(\eta)\right)+2 \lambda S_{v_{1}}^{*^{\prime}}(\eta)\right\} \\
\quad-2 \eta \lambda\left[1-(1-p) S_{v_{1}}^{*}(\eta)-p S_{v_{1}}^{*}(\eta) S_{v_{2}}^{*}(\eta)\right] \\
\quad+2\left(\lambda^{2}\left(1-z_{1}\right)+\eta^{2}\right)\left\{1+\lambda(1-p) S_{v_{1}}^{*^{\prime}}(\eta)+p \lambda\left(S_{v_{1}}^{*^{\prime}}(\eta) S_{v_{2}}^{*}(\eta)+\right.\right.
\end{aligned}
\end{aligned}
$$




$$
\begin{aligned}
& \left.\left.S_{v_{1}}^{*}(\eta) S_{v_{2}}^{*^{\prime}}(\eta)\right)\right\} N_{5}^{\prime}(1)=-p \lambda E\left(S_{b_{2}}\right) \\
& N_{5}^{\prime \prime}(1)=-p\left[2 \lambda E\left(S_{b_{2}}\right)+\lambda^{2} E\left(S_{b_{2}}^{2}\right)\right] \\
& N_{6}^{\prime}(1)=\left[\eta \lambda+\eta^{2}+\lambda^{2}\left(1-z_{1}\right)\right]\left[1-(1-p) S_{v_{1}}^{*}(\eta)-p S_{v_{1}}^{*}(\eta) S_{v_{2}}^{*}(\eta)\right] \\
& -\eta \lambda^{2}\left(1-z_{1}\right) E\left(S_{b_{1}}\right) S_{v_{1}}^{*}(\eta)\left(1-S_{v_{2}}^{*}(\eta)\right)-\eta \lambda\left(1-z_{1}\right) S_{v_{1}}^{*}(\eta) S_{v_{2}}^{*}(\eta) \\
& N_{6}^{\prime \prime}(1)=2 \eta \lambda\left\{\left[1-\lambda(1-p) E\left(S_{b_{1}}\right)\right] S_{v_{1}}^{*}(\eta)\left(1-S_{v_{2}}^{*}(\eta)\right)-p S_{v_{1}}^{*}(\eta)\left(1-S_{v_{2}}^{*}(\eta)\right)\right. \\
& +(1-p) \lambda S_{v_{1}}^{*^{\prime}}(\eta)+p \lambda\left(S_{v_{1}}^{*^{\prime}}(\eta) S_{v_{2}}^{*}(\eta)+S_{v_{1}}^{*}(\eta) S_{v_{2}}^{*^{\prime}}(\eta)\right)+\lambda E\left(S_{b_{1}}\right)(1- \\
& \left.\left.S_{v_{1}}^{*}(\eta)\right)\right\} \\
& +\eta \lambda\left(1-z_{1}\right)\left\{p \lambda^{2} E\left(S_{b_{1}}^{2}\right)+2 \lambda S_{v_{1}}^{*^{\prime}}(\eta) S_{v_{2}}^{*}(\eta)+2 \lambda^{2}(1-p) S_{v_{1}}^{*^{\prime}}(\eta) E\left(S_{b_{1}}\right)(1-\right. \\
& \left.S_{v_{2}}^{*}(\eta)\right) \\
& \left.+2 \lambda E\left(S_{b_{1}}\right)\left(1-S_{v_{1}}^{*}(\eta)\right)-\lambda^{2} E\left(S_{b_{1}}^{2}\right) S_{v_{1}}^{*}(\eta)+2 \lambda^{2} E\left(S_{b_{1}}\right) S_{v_{1}}^{*^{\prime}}(\eta)\right\} \\
& +\left[1-(1-p) S_{v_{1}}^{*}(\eta)-p S_{v_{1}}^{*}(\eta) S_{v_{2}}^{*}(\eta)\right]\left\{2 \eta^{2} \lambda E\left(S_{b_{1}}\right)-\lambda^{3}\left(1-z_{1}\right) \eta E\left(S_{b_{1}}^{2}\right)\right. \\
& \left.+2 \lambda^{3}\left(1-z_{1}\right) E\left(S_{b_{1}}\right)\right\}+2\left[\eta^{2}+\lambda^{2}\left(1-z_{1}\right)-\lambda^{2} \eta\left(1-z_{1}\right) E\left(S_{b_{1}}\right)\right] \\
& \times\left\{1+\lambda(1-p) S_{v_{1}}^{*}(\eta)+p \lambda\left(S_{v_{1}}^{*^{\prime}}(\eta) S_{v_{2}}^{*}(\eta)+S_{v_{1}}^{*}(\eta) S_{v_{2}}^{*^{\prime}}(\eta)\right)\right\} \\
& D_{1}^{\prime}(1)=-\lambda\left[1-(1-p) S_{v_{1}}^{*}(\eta)-p S_{v_{1}}^{*}(\eta) S_{v_{2}}^{*}(\eta)\right] \\
& D_{1}^{\prime \prime}(1)=-2 \lambda\left[1+\lambda(1-p) S_{v_{1}}^{*^{\prime}}(\eta)+p \lambda\left(S_{v_{1}}^{*^{\prime}}(\eta) S_{v_{2}}^{*}(\eta)+S_{v_{1}}^{*}(\eta) S_{v_{2}}^{*^{\prime}}(\eta)\right)\right] \\
& D_{2}^{\prime}(1)=\eta\left[1-\lambda E\left(S_{b_{1}}\right)-p \lambda E\left(S_{b_{2}}\right)\right] \\
& D_{2}^{\prime \prime}(1)=-2 \lambda\left[1-\lambda E\left(S_{b_{1}}\right)-p \lambda E\left(S_{b_{2}}\right)\right]-\eta \lambda^{2}\left[E\left(S_{b_{1}}^{2}\right)+p E\left(S_{b_{2}}^{2}\right)+2 p E\left(S_{b_{1}}\right) E\left(S_{b_{2}}\right)\right]
\end{aligned}
$$

where $E\left(S_{b_{1}}\right)$ and $E\left(S_{b_{2}}\right)$ are the mean service times of stage 1 and stage 2 respectively and $E\left(S_{b_{1}}^{2}\right)$ and $E\left(S_{b_{2}}^{2}\right)$ are the second moments of the service times of stage 1 and stage 2 respectively.

\section{Particular Cases}

Case i: If no customer receives the additional second stage of service and the server never takes a vacation then on setting $p=0$ and $\eta \rightarrow \infty$ in (36) we get

$$
P(z)=\frac{\left(1-\lambda E\left(S_{b_{1}}\right)\right)(1-z) S_{b_{1}}^{*}(\lambda-\lambda z)}{S_{b_{1}}^{*}(\lambda-\lambda z)-z}
$$

Equation (39) is well known generating function of the steady state queue length distribution of an $M / G / 1$ queue irrespective of the notations.(Medhi[9]) 
Caseii : If the server never takes a vacation then taking limit as $\eta \rightarrow \infty$ in (36) we get

$$
P(z)=\frac{\left[z\left[S_{b_{1}}^{*}(\lambda-\lambda z)-1\right]+p z S_{b_{1}}^{*}(\lambda-\lambda z)\left[S_{b_{2}}^{*}(\lambda-\lambda z)-1\right]\right]}{z-(1-p) S_{b_{1}}^{*}(\lambda-\lambda z)-p S_{b_{1}}^{*}(\lambda-\lambda z) S_{b_{2}}^{*}(\lambda-\lambda z)} \times P_{0}
$$

where

$$
P_{0}=1-\lambda\left[E\left(S_{b_{1}}\right)+p E\left(S_{b_{2}}\right)\right]
$$

Equation (40) is well known generating function of the steady state queue length distribution of an $M / G / 1$ queue with second optional service studied by Madan (2000) irrespective of the notations.

\section{References}

[1] Aftab Begum. M.I., Analysis of the batch arrival $M^{X} / G / 1$ queue with exponentially distributed Multiple Working Vacations, International Journal of Mathematical Sciences and Applications, Vol.1 No.2 (2011) $865-880$.

[2] Baba. Y, On the $M^{X} / G / 1$ Queue with vacation time, Operations Research Letters, Vol.5 No.2 (1986).

[3] Gautam Choudhury., Tadj. L., Paul. M., Steady state analysis of an $M^{X} / G / 1$ queue with two phases of service and Bernoulli vacation schedule under multiple vacation policy, Appl. Math. Modelling 31(2007)1079-1091.

[4] Cooper. R.B., Introduction to Queueing Theory, Elsevier, North-Holland, New York, 2nd Edition, (1981).

[5] Doshi. B.T., Single server queues with vacations, H. Takagi (Ed.) Stochastic Analysis of the Computer and Communications Systems, North-Holland Elsevier, Amsterdam (1990). 
[6] Gross, C. and Harris, C.M., The Fundamentals of Queueing Theory, Second Edition, John Wiley and Sons, New york, 1985.

[7] Kalyanaraman. R, and Pazhani Bala Murugan. S., $M / G / 1$ queue with second optional service and with server vacation, Annamalai University Science Journal, 45 (2008) 129-134.

[8] Levy. Y, and Yechali., Utilization of the idle time in an $M / G / 1$ queuing system. Mgmt.Sc., 22 (1975) 202-211.

[9] Medhi. J., Stochastic Processes, Wiley Eastern, (1982).

[10] Madan Kailash. C., An $M / G / 1$ queue with second optional service, Queueing Systems, 34 (2000) 37-46.

[11] Madan,K.C., On a single server vacation queue with two stage heterogeneous service and deterministic server vacations, International Journal of Systems Science, 32 (2001) 837-844.

[12] Madan, Kailash C.,Gautam Choudhury.A single server queue with two phases of heterogeneous service under Bernoulli schedule and a generalized vacation time, Int.J.Inform.Manage.Sci., 16(2) (2005) 1-16.

[13] Servi. L.D., and Finn. S.G., $M / M / 1$ queues with Working Vacations (M/M/1/WV), Performance Evaluation., 50 (2002) 41-52.

[14] Teghem. J., Control of the service process in a queueing system, Eur. J. Op. Res., 23 (1986) 141-158.

[15] Thangaraj. V., $M / G / 1$ Queue with two-stage Heterogeneous service compulsory server vacation and random breakdowns, Int. J. Contemp. Math. Sci., 5 (2010) 307-322.

[16] Tian.N.,Zhao.X.,and Wang.K.,The $M / M / 1$ queue with single working vacation, International Journal of information and Management Sciences., Vol 4 (2008) 621-634. 
[17] Wu. D. and Takagi. H., The $M / G / 1$ queue with multiple working vacations, Performance Evaluations, 63 (2006) 654-681.

Received: April 4, 2013 